\title{
One-loop background calculations in the general field theory.
}

\author{
Petr I.Pronin * and Konstantin V.Stepanyantz ${ }^{\dagger}$ \\ Moscow State University, Physical Faculty, \\ Department of Theoretical Physics. \\ 117234, Moscow, Russian Federation
}

\begin{abstract}
We present master formulas for the divergent part of the one-loop effective action for a minimal operator of any order in the 4-dimensional curved space and for an arbitrary nonminimal operator in the flat space.
\end{abstract}

11.10.Gh, 04.62.+v

*E-mail:pronin@theor.phys.msu.su

$\dagger$ E-mail:stepan@theor.phys.msu.su 


\section{INTRODUCTION.}

Progress in the quantum field theory and quantum gravity in particular depends much on the development of methods for the calculation of the effective action. For a lot of problems the analyses can be confined to the one-loop approximation. In this case the effective action can be expressed as a functional trace of an elliptic operator logarithm [1]. The calculation of its divergent part in the dimensional regularization is then reduced to finding $a_{2}$ coefficient of the heat kernel expansion [2 4]. It can be made in the frames of some approaches, for example, by the proper-time method proposed by Schwinger [5] and extended to the curved space-time by DeWitt [6]. It allows to consider the simplest minimal second order operator

$$
D_{1 i}{ }^{j}=\nabla^{\mu} \nabla_{\mu} \delta_{i}{ }^{j}+S^{\mu}{ }_{i}{ }^{j} \nabla_{\mu}+W_{i}{ }^{j}
$$

On the other hand, the effective action is the sum of one-particle irreducible Feynman graphs. Explicitly covariant perturbation theory can be constructed if only the propagator

depends on the background metric. Then the calculation of divergent graphs can be made by the generalized Schwinger-DeWitt technick presented in [7]. Using this approach Barvinsky and Vilkovisky found the effective action for the theories with the operators

$$
\begin{aligned}
& D_{2{ }_{i}{ }^{j}}=\left(\nabla^{\mu} \nabla_{\mu}\right)^{2} \delta_{i}{ }^{j}+S^{\mu \nu \alpha}{ }_{i}{ }^{j} \nabla_{\mu} \nabla_{\nu} \nabla_{\alpha}+W_{i}^{\mu \nu}{ }_{i}^{j} \nabla_{\mu} \nabla_{\nu} \\
& +N^{\mu}{ }_{i}{ }^{j} \nabla_{\mu}+M_{i}{ }^{j} \\
& D_{3 \alpha}{ }^{\beta}=\nabla^{\mu} \nabla_{\mu} \delta_{\alpha}{ }^{\beta}-\lambda \nabla_{\alpha} \nabla^{\beta}+P_{\alpha}{ }^{\beta} .
\end{aligned}
$$

The method has been extended to multi-loop orders [8,9], non-Riemannian manifolds 10 14 and theories with fermions [15,16]. Some papers were devoted to varies changes in the technick of calculations 12 14.

Recently the approach proposed in [7] was applied for the calculation of non-local contributions to the effective action on the Riemannian manifold [17.

In this paper we construct the explicit expression for the divergent part of the oneloop effective action without any restrictions to the form and the order of the operator $D$. 
(We consider only theories in the dimension 4). For this purpose we use the generalized t'Hooft-Veltman approach [18].

Our paper is organized as follows.

In Sec. 2 we briefly describe the calculation of the one-loop effective action by gaussian integration and introduce some notations.

In Sec. 3 we remind t'Hooft-Veltman diagramic technick and derive Feynman rules for arbitrary minimal and nonminimal operators. We then found all divergent graphs in the flat space and calculate their degree of divergence.

In Sec. 4 we consider a theory with an arbitrary minimal operator in the curved spacetime. The main result here is an explicit expression for the one-loop contribution to the divergent part of the effective action.

Section 5 is devoted to the derivation of a master formula for an arbitrary nonminimal operator on the flat background.

In Sec. 6 we consider some particular cases and show the agreement of our formulas with the earlier known results. We find also the effective action for theories with sixth and eighth order minimal operators.

In Sec. 7 we give a brief discussion and summary of our results.

In Appendix A we describe a method to calculate the divergent part of Feynman integrals.

In Appendix B we derive some rules for handling the integration over angles in the case of a minimal operator.

\section{BACKGROUND FIELD METHOD IN THE ONE-LOOP APPROXIMATION}

Let us consider the generating functional for connected Green's functions

$$
\exp \left(\frac{i}{\hbar} W[J]\right)=\int D \varphi \exp \left(\frac{i}{\hbar}(S[\varphi]+J \varphi)\right)
$$

(In this section $\hbar \neq 1$.)

Its loop expansion may be obtained by the method of saddle-point integration. The saddle point $\varphi_{0}[J]$ satisfy 


$$
\left.\frac{\delta S}{\delta \varphi}\right|_{\varphi=\varphi_{0}}=-J
$$

Expanding $S$ about $\varphi_{0}$, we found that

$$
W[J]=S\left[\varphi_{0}\right]+J \varphi_{0}+\frac{i}{2} \hbar \operatorname{tr} \ln \frac{\delta^{2} S}{\delta \varphi_{0}^{2}}+O\left(\hbar^{2}\right) .
$$

The effective action $\Gamma[\varphi]$ is defined by making Legender transformation

$$
\Gamma[\varphi]=W[J]-\left.J \varphi\right|_{J=J[\varphi]}
$$

Substituting (5) into (6) we obtain the loop expansion of the effective action to one-loop order [1]

$$
\Gamma[\varphi]=S[\varphi]+\frac{i}{2} \hbar \operatorname{tr} \ln \frac{\delta^{2} S}{\delta \varphi^{2}}+O\left(\hbar^{2}\right) .
$$

If $\varphi$ is a complex field of an arbitrary tensor structure, the effective action takes the form

$$
\Gamma^{(1)}=i \hbar \operatorname{tr} \ln \frac{\delta^{2} S}{\delta \varphi^{* i} \delta \varphi_{j}}
$$

where latin letters denote the whole set of $\varphi$ indexes.

$$
D_{i}^{j} \equiv \frac{\delta^{2} S}{\delta \varphi^{* i} \delta \varphi_{j}}
$$

is a differential operator depending on the background field $\varphi$. Its most general form is

$$
\begin{aligned}
& D_{i}{ }^{j}=K^{\mu_{1} \mu_{2} \ldots \mu_{L}{ }_{i}^{j}} \nabla_{\mu_{1}} \nabla_{\mu_{2}} \ldots \nabla_{\mu_{L}} \\
& +S^{\mu_{1} \mu_{2} \ldots \mu_{L-1}{ }_{i}{ }^{j}} \nabla_{\mu_{1}} \nabla_{\mu_{2}} \ldots \nabla_{\mu_{L-1}} \\
& +W^{\mu_{1} \mu_{2} \ldots \mu_{L-2}{ }_{i}{ }^{j}} \nabla_{\mu_{1}} \nabla_{\mu_{2}} \ldots \nabla_{\mu_{L-2}} \\
& +N^{\mu_{1} \mu_{2} \ldots \mu_{L-3}{ }_{i}{ }^{j}} \nabla_{\mu_{1}} \nabla_{\mu_{2}} \ldots \nabla_{\mu_{L-3}} \\
& +M^{\mu_{1} \mu_{2} \ldots \mu_{L-4}{ }_{i}{ }^{j}} \nabla_{\mu_{1}} \nabla_{\mu_{2}} \ldots \nabla_{\mu_{L-4}}+\ldots
\end{aligned}
$$

where $\nabla_{\mu}$ is a covariant derivative:

$$
\begin{aligned}
& \nabla_{\alpha} T^{\beta}{ }_{i}{ }^{j}=\partial_{\alpha} T^{\beta}{ }_{i}{ }^{j}+\Gamma_{\alpha \gamma}^{\beta} T^{\gamma}{ }_{i}{ }^{j} \\
& +\omega_{\alpha i}{ }^{k} T_{k}^{\beta}{ }^{j}-T^{\beta}{ }_{i}{ }^{k} \omega_{\alpha k}{ }^{j}, \\
& \nabla_{\mu} \Phi_{i}=\partial_{\mu} \Phi_{i}+\omega_{\mu i}{ }^{j} \Phi_{j} .
\end{aligned}
$$


Here $\Gamma_{\mu \nu}^{\alpha}$ is the Cristoffel symbol

$$
\Gamma_{\mu \nu}^{\alpha}=\frac{1}{2} g^{\alpha \beta}\left(\partial_{\mu} g_{\nu \beta}+\partial_{\nu} g_{\mu \beta}-\partial_{\beta} g_{\mu \nu}\right)
$$

and $\omega_{\mu i}{ }^{j}$ is a connection in the principle bundle.

Commuting covariant derivatives we can always make $K, S, W, N, M, \ldots$ symmetric in the greek indexes. This condition is very convenient for the calculations, so we will assume it to be satisfied.

The operator is called minimal if $L=2 l$ and

$$
K^{\mu \nu \ldots \alpha{ }_{i}{ }^{2}}=K_{0}{ }^{\mu \nu \ldots \alpha} \delta_{i}{ }^{j}
$$

where $K_{0}$ is a totally symmetric tensor, built by $g_{\mu \nu}$ :

$$
K_{0}{ }^{\mu \nu \alpha \beta \ldots}=\frac{1}{(2 l-1) ! !}\left(g_{\mu \nu} g_{\alpha \beta} \ldots+g_{\mu \alpha} g_{\nu \beta} \ldots+\ldots\right)
$$

(Here the sum is over all possible index replacements). If an operator can not be reduced to this form, we will call it nonminimal.

Commuting covariant derivatives we can rewrite a minimal operator in another form:

$$
\begin{aligned}
& D_{i}{ }^{j}=\delta_{i}{ }^{j} \square^{l}+S^{\mu_{1} \mu_{2} \ldots \mu_{2 l-1}{ }_{i}{ }^{j}} \nabla_{\mu_{1}} \nabla_{\mu_{2}} \ldots \nabla_{\mu_{2 l-1}} \\
& +W^{\mu_{1} \mu_{2} \ldots \mu_{2 l-2}{ }_{i}{ }^{j}} \nabla_{\mu_{1}} \nabla_{\mu_{2}} \ldots \nabla_{\mu_{2 l-2}} \\
& +N^{\mu_{1} \mu_{2} \ldots \mu_{2 l-3}{ }_{i}{ }^{j}} \nabla_{\mu_{1}} \nabla_{\mu_{2}} \ldots \nabla_{\mu_{2 l-3}} \\
& +M^{\mu_{1} \mu_{2} \ldots \mu_{2 l-4}{ }_{i}{ }^{j}} \nabla_{\mu_{1}} \nabla_{\mu_{2}} \ldots \nabla_{\mu_{2 l-4}}+\ldots
\end{aligned}
$$

where $\square \equiv \nabla_{\mu} \nabla^{\mu}$.

\section{DIAGRAMIC APPROACH IN THE BACKGROUND FIELD METHOD}

We will calculate the divergent part of the one-loop effective action

$$
\Gamma^{(1)}=\frac{i}{2} \operatorname{tr} \ln D_{i}^{j}
$$


for both arbitrary minimal and arbitrary nonminimal operators by the diagramic technick.

In order to present (16) as a sum of one-loop diagrams we separate a differential operator into a term with the largest number of derivatives plus a perturbation term:

$$
D_{i}{ }^{j}=\partial^{2 l} \delta_{i}{ }^{j}+V_{i}^{j}
$$

(minimal operator)

$$
D_{i}^{j}=K^{\mu \nu \ldots \alpha}{ }_{i}^{j} \partial \mu \partial_{\nu} \ldots \partial_{\alpha}+V_{i}^{j}
$$

(nonminimal operator)

where

$$
\begin{aligned}
& V=S^{\mu_{1} \ldots \mu_{L-1}} \partial_{\mu_{1}} \ldots \partial_{\mu_{L-1}} \\
& +W^{\mu_{1} \ldots \mu_{L-2}} \partial_{\mu_{1}} \ldots \partial_{\mu_{L-2}} \\
& +N^{\mu_{1} \ldots \mu_{L-3}} \partial_{\mu_{1}} \ldots \partial_{\mu_{L-3}} \\
& +M^{\mu_{1} \ldots \mu_{L-4}} \partial_{\mu_{1}} \ldots \partial_{\mu_{L-4}}+\ldots+O(h, \omega) .
\end{aligned}
$$

Terms $O(h, \omega)$ can be found by a series expansion of the operator in powers of $h_{\mu \nu}=g_{\mu \nu}-\eta_{\mu \nu}$ and $\omega_{\mu i}{ }^{j}$.

Then the one-loop effective action (16), say, for a minimal operator can be written as

$$
\begin{aligned}
\Gamma^{(1)} & =\frac{i}{2} \operatorname{tr} \ln D_{i}^{j}=\frac{i}{2} \operatorname{tr} \ln \left(\partial^{2 l}+V\right) \\
& =\frac{i}{2} \operatorname{tr} \ln \partial^{2 l}+\frac{i}{2} \operatorname{tr} \ln \left(1+\frac{1}{\partial^{2 l}} V\right) .
\end{aligned}
$$

First term in (20) is an infinite numeric constant and can be omitted. Expanding logarithm in the second term in powers of $V$ we obtain

$$
\begin{aligned}
\Gamma^{(1)}=\frac{i}{2} \operatorname{tr} \ln & \left(1+\frac{1}{\partial^{2 l}} V\right) \\
& =\frac{i}{2} \operatorname{tr} \sum_{k=1}^{\infty} \frac{1}{k}\left(-\frac{1}{\partial^{2 l}} V\right)^{k} .
\end{aligned}
$$

(21) can be presented as a sum of one-loop diagrams with $k$ vertexes. In the momentum space the propagator has the form $\delta_{i}^{j} / k^{2 l}$. Vertexes in the flat space are presented at the 
Fig. 1. Their expressions are rather evident, for example, the vertex with $S$-external line can be written as

$$
S^{\mu_{1} \mu_{2} \ldots \mu_{L-1}} k_{\mu_{1}} k_{\mu_{2}} \ldots k_{\mu_{L-1}} \equiv(S k)
$$

Similar notations we will use for other expressions, for example,

$$
\begin{aligned}
& (W(k+p))^{\alpha} \equiv W^{\mu \nu \ldots \beta \alpha}(k+p)_{\mu}(k+p)_{\nu} \ldots(k+p)_{\beta} \\
& (S k)^{\alpha \beta} \equiv S^{\mu \nu \ldots \gamma \alpha \beta} k_{\mu} k_{\nu} \ldots k_{\gamma} .
\end{aligned}
$$

Numerical factors for the Feynman graphs can be easily found by (21).

The number of diagrams in (21) is infinite, but most of them are convergent. Really, it is easy to see that the degree of divergence of a one-loop graph with $s$-vertexes, $w$ $W$-vertexes, $n N$-vertexes, $m M$-vertexes and so on $(k=s+w+n+m+\ldots)$ in the flat space $\left(h_{\mu \nu}=0, \omega_{\mu i}{ }^{j}=0\right)$ is

$$
I=4-s-2 w-3 n-4 m-\ldots
$$

Therefore, there are only a finite number of the divergent diagrams. They are presented at the Fig. 2. (We excluded divergent graphs, that give zero contribution to the effective action.)

(24) is valid also for a nonminimal operator. In this case the propagator takes the form

$$
\frac{1}{K^{\mu_{1} \ldots \mu_{L}}{ }^{j} \partial_{\mu_{1}} \ldots \partial_{\mu_{L}}}
$$

in the coordinate space, or $(K k)^{-1}{ }_{i}^{j}$ in the momentum space, where

$$
\begin{aligned}
& (K k)_{i}{ }^{j} \equiv K_{i}^{\mu \nu \ldots \alpha}{ }_{i}^{j} k_{\mu} k_{\nu} \ldots k_{\alpha}, \\
& (K k)^{-1}{ }_{i}^{m}(K k)_{m}{ }^{j}=\delta_{i}{ }^{j} .
\end{aligned}
$$

We will assume that $(K k)^{-1}{ }_{i}{ }^{j}$ exists. It can be made by fixing gauge invariances. 


\section{EFFECTIVE ACTION FOR A MINIMAL OPERATOR}

\section{A. Flat space}

Now we should calculate diagrams presented at the Fig. 2. We will do it using dimensional regularization. So, in order to find the divergent part of an integral

$$
\int d^{d} k f(k, p)
$$

it is necessary to expand the function $f$ into series, retain only logarithmically divergent terms and perform the integration in remaining integrals

$$
\int d^{d} k \frac{1}{k^{M+4}} k_{\mu_{1}} k_{\mu_{2}} \ldots k_{\mu_{M}}
$$

according to the following equations

$$
\begin{aligned}
& \int d^{d} k \frac{1}{k^{2 m+5}} k_{\mu_{1}} k_{\mu_{2}} \ldots k_{\mu_{2 m+1}}=0 \\
& \int d^{d} k \frac{1}{k^{2 m+4}} k_{\mu_{1}} k_{\mu_{2}} \ldots k_{\mu_{2 m}} \\
& =-\frac{2 i \pi^{2}}{(d-4)}<n_{\mu_{1}} n_{\mu_{2}} \ldots n_{\mu_{2 m}}>
\end{aligned}
$$

where

$$
\begin{aligned}
& <n_{\mu_{1}} n_{\mu_{2}} \ldots n_{\mu_{2 m}}>\equiv \frac{1}{2^{m}(m+1) !} \\
& \times\left(g_{\mu_{1} \mu_{2}} g_{\mu_{3} \mu_{4}} \ldots g_{\mu_{2 m-1} \mu_{2 m}}\right. \\
& \left.\quad+g_{\mu_{1} \mu_{3}} g_{\mu_{2} \mu_{4}} \ldots g_{\mu_{2 m-1} \mu_{2 m}}+\ldots\right)
\end{aligned}
$$

is a result of the integration over angles (for more details see appendix A).

(The sum in the equation (28) is over all possible index replacements)

Let us consider first logarithmically divergent graphs (2a)-(2e). In order to find the divergent part of the diagram in this case we should retain only terms without external mo-

mentums and perform the remaining integration. As an example let us calculate a diagram $(2 \mathrm{~d})$. 


$$
(2 d)=\frac{i}{2(2 \pi)^{4}} \operatorname{tr} \int d^{d} k \frac{(S k)(N(k-p))}{k^{2 l}(k-p)^{2 l}} .
$$

According to the previous discussion we can easily conclude that

$$
\begin{aligned}
(2 d)_{\infty}=\frac{i}{2(2 \pi)^{4}} & \int d^{d} k \frac{1}{k^{4}} \operatorname{tr}<\hat{S} \hat{N}> \\
& =\frac{1}{16 \pi^{2}(d-4)} \operatorname{tr}<\hat{S} \hat{N}>
\end{aligned}
$$

where

$$
\begin{aligned}
& \hat{S} \equiv(K n)^{-1}(S n), \\
& \hat{N} \equiv(K n)^{-1}(N n),
\end{aligned}
$$

and $n_{\mu}=k_{\mu} / \sqrt{k^{\alpha} k_{\alpha}}$ is a unit vector. In particular, for a minimal operator

$$
\begin{aligned}
& \hat{S}=(S n)=S^{\mu \nu \ldots \alpha} n_{\mu} n_{\nu} \ldots n_{\alpha} \\
& \hat{N}=(N n)=N^{\mu \nu \ldots \alpha} n_{\mu} n_{\nu} \ldots n_{\alpha} .
\end{aligned}
$$

In a similar fashion we have

$$
\begin{aligned}
& (2 a)_{\infty}=\frac{1}{64 \pi^{2}(d-4)} \operatorname{tr}<\hat{S}^{4}>, \\
& (2 b)_{\infty}=-\frac{1}{16 \pi^{2}(d-4)} \operatorname{tr}<\hat{W} \hat{S}^{2}>, \\
& (2 c)_{\infty}=\frac{1}{32 \pi^{2}(d-4)} \operatorname{tr}<\hat{W}^{2}>, \\
& (2 e)_{\infty}=-\frac{1}{16 \pi^{2}(d-4)} \operatorname{tr}<\hat{M}>.
\end{aligned}
$$

The calculation of linearly divergent graphs is a bit more difficult. For example, in order to find the divergent part of the diagram

$$
(2 g)=\frac{i}{2(2 \pi)^{4}} \operatorname{tr} \int d^{d} k \frac{(S k)(W(k-p))}{k^{2 l}(k-p)^{2 l}}
$$

we should retain only terms linear in external momentum $p$. Using the rule (B5) we obtain

$$
\begin{aligned}
\frac{1}{16 \pi^{2}(d-4)} \operatorname{tr}<2 l(p n) \hat{S} \hat{W}-(2 l-2) p_{\mu} \hat{W}^{\mu} \hat{S}> \\
\\
=\frac{1}{32 \pi^{2}(d-4)} \operatorname{tr}<(2 l-1) p_{\mu} \hat{S}^{\mu} \hat{W}-(2 l-2) p_{\mu} \hat{S} \hat{W}^{\mu}>
\end{aligned}
$$


After a substitution $p_{\mu} \hat{S} \rightarrow-\partial_{\mu} \hat{S}$, the result for this diagram takes the form

$$
(2 g)_{\infty}=\frac{1}{32 \pi^{2}(d-4)} t r<-(2 l-1) \partial_{\mu} \hat{S}^{\mu} \hat{W}+(2 l-2) \partial_{\mu} \hat{S} \hat{W}^{\mu}>.
$$

The second linearly divergent graph

$$
(2 h)=-\frac{i}{6(2 \pi)^{4}} \operatorname{tr} \int d^{d} k \frac{\underset{(-p)}{(S k)(-q)}(\underset{(k+q)}{(\underset{(p+q)}{S(k-p)})}}{k^{2 l}(k-p)^{2 l}(k+q)^{2 l}}
$$

can be calculated in the same way. (Here indexes in the bottom point the argument of $S$ ). The answer is

$$
(2 h)_{\infty}=\frac{(2 l-1)}{48 \pi^{2}(d-4)} \operatorname{tr}<\partial_{\mu} \hat{S}^{\mu} \hat{S} \hat{S}-\partial_{\mu} \hat{S} \hat{S} \hat{S}^{\mu}>
$$

So, we should consider only the rest quadratically divergent diagram.

$$
(2 f)=\frac{i}{4(2 \pi)^{4}} \operatorname{tr} \int d^{d} k \frac{(S k)(S(k-p))}{k^{2 l}(k-p)^{2 l}} .
$$

Retaining logarithmically divergent terms we can easily find that

$$
\begin{aligned}
(2 f)_{\infty}=\frac{1}{16 \pi^{2}(d-4)} \operatorname{tr} \int d^{4} x<\frac{l^{2}}{2(2 l+1)} \partial_{\mu} \hat{S} \partial^{\mu} \hat{S}+ & \frac{(2 l-1)^{2} l}{4(2 l+1)} \partial_{\mu} \hat{S}^{\mu} \partial_{\nu} \hat{S}^{\nu} \\
& -\frac{(2 l-1)\left(l^{2}-1\right)}{2(2 l+1)} \partial_{\mu} \hat{S}^{\mu \nu} \partial_{\nu} \hat{S}>.
\end{aligned}
$$

Collecting the results (30)-(40) we obtain the divergent part of the one-loop effective action for the minimal operator (15) in the flat space:

$$
\begin{aligned}
& \left(\Gamma_{\infty}^{(1)}\right)^{\text {flat }}=\frac{1}{16 \pi^{2}(d-4)} \operatorname{tr} \int d^{4} x<\frac{l^{2}}{2(2 l+1)} \partial_{\mu} \hat{S} \partial^{\mu} \hat{S}-\frac{(2 l-1)\left(l^{2}-1\right)}{2(2 l+1)} \partial_{\mu} \hat{S}^{\mu \nu} \partial_{\nu} \hat{S} \\
& +\frac{(2 l-1)^{2} l}{4(2 l+1)} \partial_{\mu} \hat{S}^{\mu} \partial_{\nu} \hat{S}^{\nu}+\frac{(2 l-1)}{3} \partial_{\mu} \hat{S}^{\mu} \hat{S} \hat{S}-\frac{(2 l-1)}{3} \partial_{\mu} \hat{S} \hat{S} \hat{S}^{\mu}+(l-1) \partial_{\mu} \hat{S} \hat{W}^{\mu} \\
& -\frac{(2 l-1)}{2} \partial_{\mu} \hat{S}^{\mu} \hat{W}+\hat{S} \hat{N}+\frac{1}{2} \hat{W}^{2}+\frac{1}{4} \hat{S}^{4}-\hat{W} \hat{S}^{2}-\hat{M}>.
\end{aligned}
$$

\section{B. Extension to the curved space}

In order to obtain the divergent part of a one-loop effective action in the curved space we consider a minimal operator in the form (15). 
In this case we can not calculate all divergent graphs, because their number is infinite. (The matter is that the degree of divergence does not depend on the number of $h_{\mu \nu}$ vertexes and there are infinite number of such vertexes too). Nevertheless if we note that the answer should be invariant under the general coordinate transformations, the result can be found by calculating only a finite number of graphs. Really, we should replace derivatives in (41) by the covariant ones and add expressions, containing curvature tensors $R_{\alpha \mu \nu}^{\sigma}$ and $F_{\mu \nu}$. The most general form of additional terms is

$$
\begin{aligned}
& \frac{1}{16 \pi^{2}(d-4)} \operatorname{tr} \int d^{4} x \sqrt{-g}<a_{1} R^{2}+a_{2} R_{\mu \nu} R^{\mu \nu}+a_{3} \hat{W}^{\alpha \beta} R_{\alpha \beta}+a_{4} \hat{W} R+a_{5} \nabla_{\mu} \hat{S}^{\mu \alpha \beta} R_{\alpha \beta} \\
& +a_{6} \nabla_{\mu} \hat{S}^{\mu} R+a_{7} \hat{S}^{2} R+a_{8} R_{\alpha \beta} \hat{S}^{\alpha} \hat{S}^{\beta}+a_{9} R_{\alpha \beta} \hat{S}^{\alpha \beta} \hat{S}+a_{10} R_{\mu \nu \alpha \beta} \hat{S}^{\mu \alpha} \hat{S}^{\nu \beta}+a_{11} F_{\mu \nu} F^{\mu \nu} \\
& +a_{12} F_{\mu \nu} \hat{S}^{\mu} \hat{S}^{\nu}+a_{13} F_{\mu \nu} \nabla^{\mu} \hat{S}^{\nu}>.
\end{aligned}
$$

where

$$
\begin{aligned}
& R_{\beta \mu \nu}^{\alpha}=\partial_{\mu} \Gamma_{\nu \beta}^{\alpha}-\partial_{\nu} \Gamma_{\mu \beta}^{\alpha}+\Gamma_{\mu \gamma}^{\alpha} \Gamma_{\nu \beta}^{\gamma}-\Gamma_{\nu \gamma}^{\alpha} \Gamma_{\mu \beta}^{\gamma}, \\
& F_{\mu \nu i}{ }^{j}=\partial_{\mu} \omega_{\nu i}{ }^{j}-\partial_{\nu} \omega_{\mu i}{ }^{j}+\omega_{\mu i}{ }^{k} \omega_{\nu k}{ }^{j}-\omega_{\nu i}{ }^{k} \omega_{\mu k}{ }^{j}, \\
& R_{\mu \nu}=R^{\alpha}{ }_{\mu \alpha \nu}, \quad R=g^{\mu \nu} R_{\mu \nu} .
\end{aligned}
$$

(We take into account that the expression $R_{\mu \nu \alpha \beta} R^{\mu \nu \alpha \beta}-4 R_{\mu \nu} R^{\mu \nu}+R^{2}$ is a total derivative and should be omitted).

Then the coefficients $a_{1}-a_{13}$ can be found by calculating the diagrams presented at the

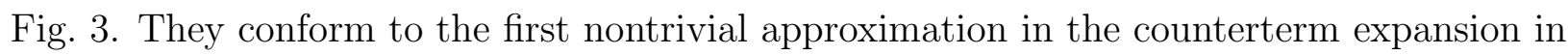
powers of weak fields $h_{\mu \nu}=g_{\mu \nu}-\eta_{\mu \nu}$ and $\omega_{\mu i}{ }^{j}$.

As an example we consider diagrams (3a) and (3b).

The vertex with $h_{\mu \nu}$ in (3a) should be found according to (19) by series expansion of $\square^{l}$ to the first order and has the form

$$
\sum_{m=0}^{l-1} k^{2 m}(k-p)^{2 l-2 m-2}\left(-h^{\mu \nu} k_{\mu}(k-p)_{\nu}+\frac{1}{2} h^{\alpha}{ }_{\alpha} p^{\mu}(k-p)_{\mu}\right) .
$$

Then the graph (3a) can be written as 


$$
\begin{aligned}
(3 a)=\frac{i}{2(2 \pi)^{4}} \operatorname{tr} \int d^{d} k & \frac{1}{k^{2 l}(k-p)^{2 l}}(W k) \\
& \times \sum_{m=0}^{l-1} k^{2 m}(k-p)^{2 l-2 m-2}\left(-h^{\mu \nu} k_{\mu}(k-p)_{\nu}+\frac{1}{2} h^{\alpha}{ }_{\alpha} p^{\mu}(k-p)_{\mu}\right) .
\end{aligned}
$$

It is easy to see that the considered diagram is quadratically divergent. So, retaining terms quadratic in external momentum $p$ we obtain the divergent part

$$
\begin{aligned}
\frac{1}{16 \pi^{2}(d-4)} \operatorname{tr}<\hat{W}\left(l(l+1)\left(n^{\gamma} p_{\gamma}\right)\left(h^{\mu \nu} n_{\mu} p_{\nu}+\frac{1}{2} h_{\alpha}^{\alpha}\left(n^{\beta} p_{\beta}\right)\right)-\right. \\
\left.-h^{\mu \nu} n_{\mu} n_{\nu}\left(-\frac{1}{2} l(l+1) p^{2}+\frac{2}{3} l(l+1)(l+2)\left(n^{\alpha} p_{\alpha}\right)^{2}\right)-\frac{l}{2} h^{\alpha}{ }_{\alpha} p^{2}\right)>.
\end{aligned}
$$

Using rules formulated in the appendix $\mathbb{B}$, it can be written as

$$
\begin{aligned}
& (3 a)_{\infty}=\frac{1}{16 \pi^{2}(d-4)} \operatorname{tr}<-\frac{1}{12}(2 l-3)(2 l-4)(2 l-5) \hat{W}^{\mu \nu \alpha \beta} h_{\alpha \beta} p_{\mu} p_{\nu} \\
& +\frac{l}{6} \hat{W}\left(-h^{\alpha}{ }_{\alpha} p^{2}+h^{\mu \nu} p_{\mu} p_{\nu}\right)-\frac{1}{12}(l-1)(2 l-3) \hat{W}^{\mu \nu}\left(-p^{2} h_{\mu \nu}-p_{\mu} p_{\nu} h_{\alpha}^{\alpha}+2 p_{\mu} p_{\alpha} h_{\nu}^{\alpha}\right)>.
\end{aligned}
$$

Nevertheless, (47) can not be presented as a weak field limit of a covariant expression. The matter is that the graphs (3a) and (3b) can not be considered separately.

The vertex in the tadpole diagram (3b) can be found by series expansion of $W^{\mu \nu \ldots \alpha_{i}{ }^{j}} \nabla_{\mu} \nabla_{\nu} \ldots \nabla_{\alpha}$ in powers of $h_{\mu \nu}$ to the first order. Then, retaining only nontrivial contributions, we have

$$
\begin{aligned}
(3 b)=\frac{i}{2(2 \pi)^{4}} & t r \int d^{d} k \frac{1}{k^{2 l}} W^{\mu_{1} \mu_{2} \ldots \mu_{2 l-2}} \\
& \times \sum_{m=0}^{2 l-4}(2 l-3-m)(k+p)_{\mu_{1}} \ldots(k+p)_{\mu_{m}} \Gamma_{\mu_{m+1} \mu_{m+2}}^{\alpha(1)} k_{\alpha} k_{\mu_{m+3}} \ldots k_{\mu_{2 l-2}},
\end{aligned}
$$

where

$$
\Gamma_{\beta \gamma}^{\alpha(1)}=\frac{1}{2}\left(p_{\beta} h_{\gamma}{ }^{\alpha}+p_{\gamma} h_{\beta}{ }^{\alpha}-p^{\alpha} h_{\beta \gamma}\right)
$$

is the Cristoffel symbol in the weak field limit.

Using (B3) after simple transformations the divergent part of (3b) can be written as

$$
\begin{aligned}
(3 b)_{\infty}=\frac{(2 l-3)}{192 \pi^{2}(d-4)(2 l-5)} \\
\times \\
\times \operatorname{tr}<\hat{W}^{\mu \nu \alpha \beta} h_{\alpha \beta} p_{\mu} p_{\nu}>.
\end{aligned}
$$


The sum of (47) and (50) unlike each of the graphs considered separately can be presented as a weak field approximation of a covariant expression

$$
\begin{aligned}
\frac{1}{16 \pi^{2}(d-4)} & \operatorname{tr} \int d^{4} x \sqrt{-g}<\frac{l}{6} \hat{W} R \\
& -\frac{1}{6}(l-1)(2 l-3) R_{\mu \nu} \hat{W}^{\mu \nu}>
\end{aligned}
$$

that is the ultimate answer for the diagrams (3a) and (3b).

The other graphs are considered in the same way. After rather cumbersome calculations we obtain the following formula for the divergent part of the one-loop effective action for a minimal operator (15) in the curved space:

$$
\begin{aligned}
& \Gamma_{\infty}^{(1)}=\frac{1}{16 \pi^{2}(d-4)} \operatorname{tr} \int d^{4} x \sqrt{-g}<-\frac{(2 l-1)\left(l^{2}-1\right)}{2(2 l+1)} \nabla_{\mu} \hat{S}^{\mu \nu} \nabla_{\nu} \hat{S}+\frac{l^{2}}{2(2 l+1)} \nabla_{\mu} \hat{S} \nabla^{\mu} \hat{S} \\
& +\frac{(2 l-1)^{2} l}{4(2 l+1)} \nabla_{\mu} \hat{S}^{\mu} \nabla_{\nu} \hat{S}^{\nu}+\frac{(2 l-1)}{3} \nabla_{\mu} \hat{S}^{\mu} \hat{S} \hat{S}-\frac{(2 l-1)}{3} \nabla_{\mu} \hat{S} \hat{S} \hat{S}^{\mu}+(l-1) \nabla_{\mu} \hat{S} \hat{W}^{\mu} \\
& -\frac{(2 l-1)}{2} \nabla_{\mu} \hat{S}^{\mu} \hat{W}+\hat{S} \hat{N}+\frac{1}{2} \hat{W}^{2}+\frac{1}{4} \hat{S}^{4}-\hat{W} \hat{S}^{2}-\hat{M}+\frac{(2 l-1)(2 l-3)(l-1)}{12} \nabla_{\mu} \hat{S}^{\mu \alpha \beta} R_{\alpha \beta} \\
& -\frac{(2 l-1) l}{12} \nabla_{\mu} \hat{S}^{\mu} R-\frac{(2 l-3)(l-1)}{6} \hat{W}^{\alpha \beta} R_{\alpha \beta}+\frac{l}{6} \hat{W} R+\frac{(2 l-1)^{2}(l+2)}{24(2 l+1)} R_{\alpha \beta} \hat{S}^{\alpha} \hat{S}^{\beta} \\
& -\frac{l}{6} \hat{S}^{2} R+\frac{(2 l-1)(l-1)}{6} R_{\alpha \beta} \hat{S}^{\alpha \beta} \hat{S}-\frac{(2 l-1)(l-1)^{2}(l+2)}{12(2 l+1)} R_{\mu \nu \alpha \beta} \hat{S}^{\mu \alpha} \hat{S}^{\nu \beta}+\frac{l}{12} F_{\mu \nu} F^{\mu \nu} \\
& +\frac{l(2 l-1)}{6} \nabla_{\mu} \hat{S}_{\nu} F^{\mu \nu}+\frac{(2 l-1)^{2}(l+1)}{4(2 l+1)} F_{\mu \nu} \hat{S}^{\mu} \hat{S}^{\nu}+l\left(\frac{1}{120} R^{2}+\frac{1}{60} R_{\mu \nu} R^{\mu \nu}\right)>.
\end{aligned}
$$

It is more convenient sometimes to use another form of the operator:

$$
\begin{aligned}
& D_{i}{ }^{j}=\delta_{i}{ }^{j} K_{0}{ }^{\mu_{1} \mu_{2} \ldots \mu_{2 l}} \nabla_{\mu_{1}} \nabla_{\mu_{2}} \ldots \nabla_{\mu_{2 l}} \\
& +S^{\mu_{1} \mu_{2} \ldots \mu_{2 l-1}{ }_{i}{ }^{j}} \nabla_{\mu_{1}} \nabla_{\mu_{2}} \ldots \nabla_{\mu_{2 l-1}} \\
& +W^{\mu_{1} \mu_{2} \ldots \mu_{2 l-2}{ }_{i}{ }^{j}} \nabla_{\mu_{1}} \nabla_{\mu_{2}} \ldots \nabla_{\mu_{2 l-2}} \\
& +N^{\mu_{1} \mu_{2} \ldots \mu_{2 l-3}{ }_{i}{ }^{j}} \nabla_{\mu_{1}} \nabla_{\mu_{2}} \ldots \nabla_{\mu_{2 l-3}} \\
& +M^{\mu_{1} \mu_{2} \ldots \mu_{2 l-4}{ }_{i}{ }^{j}} \nabla_{\mu_{1}} \nabla_{\mu_{2}} \ldots \nabla_{\mu_{2 l-4}}+\ldots,
\end{aligned}
$$

where $K_{0}$ was defined in (14).

By the help of the same method we found the following answer for the divergent part of the one-loop effective action: 


$$
\begin{aligned}
& \Gamma_{\infty}^{(1)}=\frac{1}{16 \pi^{2}(d-4)} \operatorname{tr} \int d^{4} x \sqrt{-g}<-\frac{(2 l-1)\left(l^{2}-1\right)}{2(2 l+1)} \nabla_{\mu} \hat{S}^{\mu \nu} \nabla_{\nu} \hat{S}+\frac{l^{2}}{2(2 l+1)} \nabla_{\mu} \hat{S} \nabla^{\mu} \hat{S} \\
& +\frac{(2 l-1)^{2} l}{4(2 l+1)} \nabla_{\mu} \hat{S}^{\mu} \nabla_{\nu} \hat{S}^{\nu}+\frac{(2 l-1)}{3} \nabla_{\mu} \hat{S}^{\mu} \hat{S} \hat{S}-\frac{(2 l-1)}{3} \nabla_{\mu} \hat{S} \hat{S} \hat{S}^{\mu}+(l-1) \nabla_{\mu} \hat{S} \hat{W}^{\mu} \\
& -\frac{(2 l-1)}{2} \nabla_{\mu} \hat{S}^{\mu} \hat{W}+\hat{S} \hat{N}+\frac{1}{2} \hat{W}^{2}+\frac{1}{4} \hat{S}^{4}-\hat{W} \hat{S}^{2}-\hat{M}+\frac{(2 l-1)(2 l-3)(l-1)}{6(l+1)} \nabla_{\mu} \hat{S}^{\mu \alpha \beta} R_{\alpha \beta} \\
& -\frac{l^{2}(2 l-1)}{6(l+1)} \nabla_{\mu} \hat{S}^{\mu} R+\frac{l^{2}}{3(l+1)} \hat{W} R-\frac{(2 l-3)(l-1)}{3(l+1)} \hat{W}^{\mu \nu} R_{\mu \nu}-\frac{(2 l-1)^{2}(l-4)}{24(2 l+1)} R_{\mu \nu} \hat{S}^{\mu} \hat{S}^{\nu} \\
& -\frac{l^{2}}{2(2 l+1)} \hat{S}^{2} R-\frac{(2 l-1)(l-1)^{2}(l+2)}{12(2 l+1)} R_{\mu \nu \alpha \beta} \hat{S}^{\mu \alpha} \hat{S}^{\nu \beta}+\frac{(2 l-1)(l-1)(l+2)}{6(2 l+1)} R_{\mu \nu} \hat{S}^{\mu \nu} \hat{S} \\
& +\frac{l^{2}\left(-7 l^{2}+4 l+12\right)}{540} R_{\mu \nu} R^{\mu \nu}+\frac{l^{2}\left(3 l^{2}+4 l+2\right)}{1080} R^{2}+\frac{l^{3}}{12} F^{\mu \nu} F_{\mu \nu}+\frac{(l+1)(2 l-1)^{2}}{4(2 l+1)} \hat{S}^{\mu} \hat{S}^{\nu} F_{\mu \nu} \\
& +\frac{l^{2}(2 l-1)}{3(l+1)} F^{\mu \nu} \nabla_{\mu} \hat{S}_{\nu}>.
\end{aligned}
$$

\section{NONMINIMAL OPERATOR ON THE FLAT BACKGROUND}

Let us consider a theory with an arbitrary nonminimal operator (10) in the flat space $\left(g_{\mu \nu}=\eta_{\mu \nu}, \omega_{\mu i}{ }^{j}=0\right)$.

The divergent graphs are the same as in the case of a minimal operator (see Fig. 2), but because of the difference of the propagators the calculations are also different.

As earlier, the calculation of logarithmically divergent graphs is the simplest. For example,

$$
\begin{aligned}
& (2 d)_{\infty}=\frac{i}{2(2 \pi)^{4}} \operatorname{tr} \int d^{d} k(S k)(K k)^{-1}(N(k-p)) \\
& \times\left.(K(k-p))^{-1}\right|_{\infty}=\frac{1}{16 \pi^{2}(d-4)} \operatorname{tr}<\hat{S} \hat{N}>
\end{aligned}
$$

(We should remind, that here $\hat{W}=(K n)^{-1}(W n) \neq(W n)$ and so on, see (31)).

The other logarithmically divergent graphs can be calculated in the same way. The result coincides with (33).

Now let us consider linearly divergent graphs.

$$
\begin{aligned}
(2 g)=\frac{i}{2(2 \pi)^{4}} \operatorname{tr} & \int d^{d} k(S k)(K k)^{-1} \\
& \times(W(k-p))(K(k-p))^{-1} .
\end{aligned}
$$


We should expand this expression into series over external momentum $p$ and retain logarithmically divergent terms (It is easy to see that they are linear in $p$ ).

Using an identity

$$
1_{i}{ }^{j}=(K(k+p))_{i}{ }^{m}(K(k+p))^{-1}{ }_{m}^{j},
$$

we can easily obtain that

$$
\begin{aligned}
& (K(k+p))^{-1}=(K k)^{-1}-L p_{\mu}(K k)^{-1}(K k)^{\mu}(K k)^{-1} \\
& +p_{\mu} p_{\nu}\left(-\frac{1}{2} L(L-1)(K k)^{-1}(K n)^{\mu \nu}(K k)^{-1}\right. \\
& \left.+L^{2}(K k)^{-1}(K n)^{\mu}(K k)^{-1}(K k)^{\nu}(K k)^{-1}\right)+O\left(p^{3}\right) .
\end{aligned}
$$

So, the divergent part of the diagram takes the following form

$$
\begin{aligned}
\frac{1}{16 \pi^{2}(d-4)} \operatorname{tr}<L p_{\mu} \hat{S} \hat{W} \hat{K}^{\mu} & \\
& -(L-2) p_{\mu} \hat{S} \hat{W}^{\mu}>.
\end{aligned}
$$

Making a substitution $p_{\mu} \hat{S} \rightarrow-\partial_{\mu} \hat{S}$, we found the ultimate expression in the coordinate space

$$
\begin{aligned}
(2 g)_{\infty}=\frac{1}{16 \pi^{2}(d-4)} t r< & -L \partial_{\mu} \hat{S} \hat{W} \hat{K}^{\mu} \\
& +(L-2) \partial_{\mu} \hat{S} \hat{W}^{\mu}>.
\end{aligned}
$$

In a similar fashion we find that the divergent part of the graph with three external S-lines

$$
\begin{aligned}
(2 h)=-\frac{i}{6(2 \pi)^{4}} \operatorname{tr} \int d^{d} k(S k)(K k)^{-1} & (S(k+q)) \times \\
& \times(K(k+q))^{-1}(S(k-p))(K(k-p))^{-1}
\end{aligned}
$$

is

$$
\begin{aligned}
&(2 h)_{\infty}=\frac{1}{16 \pi^{2}(d-4)} t r<\frac{1}{3}\left((L-1) \partial_{\mu} \hat{S}^{\mu} \hat{S} \hat{S}-\right. \\
&\left.-L \partial_{\mu} \hat{S} \hat{K}^{\mu} \hat{S} \hat{S}-(L-1) \partial_{\mu} \hat{S} \hat{S} \hat{S}^{\mu}+L \partial_{\mu} \hat{S} \hat{S} \hat{S} \hat{K}^{\mu}\right)>
\end{aligned}
$$


The divergent part of the diagram (2f)

$$
(2 f)=\frac{i}{4(2 \pi)^{4}} \operatorname{tr} \int d^{d} k(S k)(K k)^{-1}(S(k-p))(K(k-p))^{-1}
$$

should be calculated by series expansion in powers of $p$ to the second order. It is easy to see, that

$$
\begin{aligned}
(2 f)_{\infty}= & \frac{1}{16 \pi^{2}(d-4)} t r<-\frac{1}{2} \partial_{\mu} \hat{S} \partial_{\nu} \hat{S}\left(-\frac{1}{2} L(L-1) \hat{K}^{\mu \nu}+\right. \\
& \left.+L^{2} \hat{K}^{\mu} \hat{K}^{\nu}\right)+\frac{1}{2} L(L-1) \partial_{\mu} \hat{S} \partial_{\nu} \hat{S}^{\nu} \hat{K}^{\mu}-\frac{1}{4}(L-1)(L-2) \partial_{\mu} \hat{S} \partial_{\nu} \hat{S}^{\mu \nu}>.
\end{aligned}
$$

Collecting contributions of all graphs we obtain the following expression for the divergent part of the one-loop effective action

$$
\begin{aligned}
& \left(\Gamma_{\infty}^{(1)}\right)^{\text {flat }}=\frac{1}{16 \pi^{2}(d-4)} \operatorname{tr} \int d^{4} x<\frac{1}{4} \hat{S}^{4}-\hat{W} \hat{S}^{2}+\frac{1}{2} \hat{W}^{2}+\hat{S} \hat{N}-\hat{M}-L \partial_{\mu} \hat{S} \hat{W} \hat{K}^{\mu} \\
& +(L-2) \partial_{\mu} \hat{S} \hat{W}^{\mu}+\frac{1}{3}\left((L-1) \partial_{\mu} \hat{S}^{\mu} \hat{S} \hat{S}-L \partial_{\mu} \hat{S} \hat{K}^{\mu} \hat{S} \hat{S}-(L-1) \partial_{\mu} \hat{S} \hat{S} \hat{S}^{\mu}\right. \\
& \left.+L \partial_{\mu} \hat{S} \hat{S} \hat{S} \hat{K}^{\mu}\right)-\frac{1}{2} \partial_{\mu} \hat{S} \partial_{\nu} \hat{S}\left(-\frac{1}{2} L(L-1) \hat{K}^{\mu \nu}+L^{2} \hat{K}^{\mu} \hat{K}^{\nu}\right)+\frac{1}{2} L(L-1) \partial_{\mu} \hat{S} \partial_{\nu} \hat{S}^{\nu} \hat{K}^{\mu} \\
& -\frac{1}{4}(L-1)(L-2) \partial_{\mu} \hat{S} \partial_{\nu} \hat{S}^{\mu \nu}>.
\end{aligned}
$$

This result can be generalized to the curved space-time. It will be considered in our subsequent papers.

\section{EXAMPLES}

\section{A. The minimal second order operator}

If $l=1$ the operator (15) takes the form

$$
D_{i}{ }^{j}=\delta_{i}{ }^{j} \square+S^{\mu}{ }_{i}{ }^{j} \nabla_{\mu}+W_{i}{ }^{j} .
$$

It is easy to see that (52) gives then the following expression

$$
\begin{aligned}
& \Gamma_{\infty}^{(1)}=\frac{1}{16 \pi^{2}(d-4)} \operatorname{tr} \int d^{4} x \sqrt{-g}<\frac{1}{6} \nabla_{\mu} \hat{S} \nabla^{\mu} \hat{S}++\frac{1}{12} \nabla_{\mu} S^{\mu} \nabla_{\nu} S^{\nu}-\frac{1}{2} \nabla_{\mu} S^{\mu} W \\
& +\frac{1}{3} \nabla_{\mu} S^{\mu} \hat{S} \hat{S}-\frac{1}{3} \nabla_{\mu} \hat{S} \hat{S} S^{\mu}+\frac{1}{2} W^{2}+\frac{1}{4} \hat{S}^{4}-W \hat{S}^{2}+\frac{1}{120} R^{2}+\frac{1}{60} R_{\mu \nu} R^{\mu \nu}+\frac{1}{6} W R \\
& -\frac{1}{12} \nabla_{\mu} S^{\mu} R-\frac{1}{6} \hat{S}^{2} R+\frac{1}{24} R_{\mu \nu} S^{\mu} S^{\nu}+\frac{1}{6} S^{\mu} S^{\nu} F_{\mu \nu}+\frac{1}{6} \nabla^{\mu} S^{\nu} F_{\mu \nu}+\frac{1}{12} F^{\mu \nu} F_{\mu \nu}>.
\end{aligned}
$$


$\left(\right.$ Here $\left.\hat{W}=W, \hat{S}^{\mu}=S^{\mu}, \hat{S}=S^{\alpha} n_{\alpha}.\right)$

After substituting according to (28)

$$
\begin{aligned}
& <1>=1, \quad<n_{\mu} n_{\nu}>=\frac{1}{4} g_{\mu \nu}, \\
& <n_{\mu} n_{\nu} n_{\alpha} n_{\beta}>=\frac{1}{24}\left(g_{\mu \nu} g_{\alpha \beta}+g_{\mu \alpha} g_{\nu \beta}+g_{\mu \beta} g_{\nu \alpha}\right),
\end{aligned}
$$

we obtain the well-known result [18]:

$$
\begin{array}{r}
\Gamma_{\infty}^{(1)}=\frac{1}{16 \pi^{2}(d-4)} \operatorname{tr} \int d^{4} x \sqrt{-g}\left(\frac{1}{12} Y_{\mu \nu} Y^{\mu \nu}\right. \\
\left.+\frac{1}{2} X^{2}+\frac{1}{60} R_{\mu \nu} R^{\mu \nu}-\frac{1}{180} R^{2}\right),
\end{array}
$$

where

$$
\begin{aligned}
& Y_{\mu \nu}=\frac{1}{2} \nabla_{\mu} S_{\nu}-\frac{1}{2} \nabla_{\mu} S_{\nu}+\frac{1}{4} S_{\mu} S_{\nu}-\frac{1}{4} S_{\nu} S_{\mu}+F_{\mu \nu}, \\
& X=W-\frac{1}{2} \nabla_{\mu} S^{\mu}-\frac{1}{4} S_{\mu} S^{\mu}+\frac{1}{6} R .
\end{aligned}
$$

\section{B. The minimal forth order operator}

A minimal forth order operator $(l=2)$ has the form

$$
\begin{array}{r}
D_{i}{ }^{j}=\delta_{i}{ }^{j} \square^{2}+S_{i}^{\mu \nu \alpha}{ }_{i} \nabla_{\mu} \nabla_{\nu} \nabla_{\alpha}+W^{\mu \nu}{ }_{i}{ }^{j} \nabla_{\mu} \nabla_{\nu} \\
+N^{\mu}{ }_{i}{ }^{j} \nabla_{\mu}+M_{i}{ }^{j} .
\end{array}
$$

¿From (52) we obtained the result, that coincided with the one found in [7] by Barvinsky and Vilkovisky up to the total derivatives. (We do not presented it here because it is too large)

In particular if $S^{\mu \nu \alpha}=0$ the answer takes a rather simple form

$$
\begin{aligned}
& \Gamma_{\infty}^{(1)}=\frac{1}{16 \pi^{2}(d-4)} \operatorname{tr} \int d^{4} x \sqrt{-g}\left(\frac{1}{48} W^{2}\right. \\
& +\frac{1}{24} W^{\mu \nu} W_{\mu \nu}-M+\frac{1}{60} R^{2}+\frac{1}{30} R_{\mu \nu} R^{\mu \nu} \\
& \left.-\frac{1}{6} W^{\mu \nu} R_{\mu \nu}+\frac{1}{12} W R+\frac{1}{6} F_{\mu \nu} F^{\mu \nu}\right) .
\end{aligned}
$$




\section{The minimal sixth and eighth order operators}

Let us consider the most general sixth order operator without fifth derivatives

$$
\begin{aligned}
& D_{i}{ }^{j}=\square^{3} \delta_{i}{ }^{j}+W^{\mu \nu \alpha \beta}{ }_{i}{ }^{j} \nabla_{\mu} \nabla_{\nu} \nabla_{\alpha} \nabla_{\beta}+N^{\mu \nu \alpha}{ }_{i}{ }^{j} \nabla_{\mu} \nabla_{\nu} \nabla_{\alpha} \\
& +M^{\mu \nu}{ }_{i}{ }^{j} \nabla_{\mu} \nabla_{\nu}+P^{\mu}{ }_{i}{ }^{j} \nabla_{\mu}+Q_{i}{ }^{j} .
\end{aligned}
$$

(If $S \neq 0$ the result will be too cumbersome)

If $S=0$ the answer (52) gives

$$
\begin{aligned}
\Gamma_{\infty}^{(1)}=\frac{1}{16 \pi^{2}(d-4)} & \operatorname{tr} \int d^{4} x \sqrt{-g}<\frac{1}{2} \hat{W}^{2}-\hat{M}+\frac{l}{12} F_{\mu \nu} F^{\mu \nu}+\frac{l}{6} \hat{W} R- \\
& -\frac{1}{6}(2 l-3)(l-1) \hat{W}^{\alpha \beta} R_{\alpha \beta}+\frac{l}{120} R^{2}+\frac{l}{60} R_{\mu \nu} R^{\mu \nu}>.
\end{aligned}
$$

For the sixth order operator $l=3$, and, moreover, according to (B6) and (B7)

$$
\begin{aligned}
& <\hat{M}>=\frac{1}{4} M, \quad<\hat{W}^{\mu \nu}>=\frac{1}{4} W^{\mu \nu}, \quad<\hat{W}>=\frac{1}{8} W, \\
& <\hat{W}^{2}>=\frac{1}{80} W^{\mu \nu \alpha \beta} W_{\mu \nu \alpha \beta}+\frac{3}{80} W^{\mu \nu} W_{\mu \nu}+\frac{3}{640} W^{2},
\end{aligned}
$$

where $M \equiv M_{\mu \nu}^{\mu \nu}$ and so on.

So, for the sixth order operator we have

$$
\begin{aligned}
\Gamma_{\infty}^{(1)}= & \frac{1}{16 \pi^{2}(d-4)} \operatorname{tr} \int d^{4} x \sqrt{-g}\left(\frac{1}{160} W^{\mu \nu \alpha \beta} W_{\mu \nu \alpha \beta}+\frac{3}{160} W^{\mu \nu} W_{\mu \nu}+\right. \\
& \left.+\frac{3}{1280} W^{2}-\frac{1}{4} M-\frac{1}{4} W^{\alpha \beta} R_{\alpha \beta}+\frac{1}{16} W R+\frac{1}{40} R^{2}+\frac{1}{20} R_{\mu \nu} R^{\mu \nu}+\frac{1}{4} F_{\mu \nu} F^{\mu \nu}\right) .
\end{aligned}
$$

Eighth order operator

$$
\begin{aligned}
& D_{i}{ }^{j}=\square^{4} \delta_{i}{ }^{j}+W^{\mu \nu \alpha \beta \gamma \delta}{ }_{i}{ }^{j} \nabla_{\mu} \nabla_{\nu} \nabla_{\alpha} \nabla_{\beta} \nabla_{\gamma} \nabla_{\delta}+N^{\mu \nu \alpha \beta \gamma}{ }_{i}{ }^{j} \nabla_{\mu} \nabla_{\nu} \nabla_{\alpha} \nabla_{\beta} \nabla_{\gamma}+ \\
& +M^{\mu \nu \alpha \beta}{ }_{i}{ }^{j} \nabla_{\mu} \nabla_{\nu} \nabla_{\alpha} \nabla_{\beta}+P^{\mu \nu \alpha \alpha}{ }_{i}^{j} \nabla_{\mu} \nabla_{\nu} \nabla_{\alpha}+Q_{i}^{\mu \nu j} \nabla_{\mu} \nabla_{\nu}+T_{i}^{\mu j} \nabla_{\mu}+V_{i}{ }^{j}
\end{aligned}
$$

can be considered in a similar fashion:

In this case using rules formulated in the appendix $B$, in particular (B6) and (B7) we have 


$$
\begin{aligned}
& <\hat{M}>=\frac{1}{8} M, \quad<\hat{W}^{\mu \nu}>=\frac{1}{8} W^{\mu \nu}, \quad<\hat{W}>=\frac{5}{64} W \\
& <\hat{W}^{2}>=\frac{1}{14336}\left(16 W^{\mu \nu \alpha \beta \gamma \delta} W_{\mu \nu \alpha \beta \gamma \delta}+120 W^{\mu \nu \alpha \beta} W_{\mu \nu \alpha \beta}+\right. \\
& \left.+90 W^{\mu \nu} W_{\mu \nu}+5 W^{2}\right),
\end{aligned}
$$

and hence,

$$
\begin{aligned}
& \Gamma_{\infty}^{(1)}=\frac{1}{16 \pi^{2}(d-4)} \operatorname{tr} \int d^{4} x \sqrt{-g}\left(\frac { 1 } { 1 4 3 3 6 } \left(16 W^{\mu \nu \alpha \beta \gamma \delta} W_{\mu \nu \alpha \beta \gamma \delta}+120 W^{\mu \nu \alpha \beta} W_{\mu \nu \alpha \beta}+\right.\right. \\
& \left.\left.+90 W^{\mu \nu} W_{\mu \nu}+5 W^{2}\right)-\frac{1}{8} M-\frac{5}{16} W^{\alpha \beta} R_{\alpha \beta}+\frac{5}{96} W R+\frac{1}{30} R^{2}+\frac{1}{15} R_{\mu \nu} R^{\mu \nu}+\frac{1}{3} F_{\mu \nu} F^{\mu \nu}\right) .
\end{aligned}
$$

\section{Minimal operator as a special case of a nonminimal one}

A minimal operator can be considered as a particular case of a nonminimal one, if

$$
K_{i}^{\mu \nu \ldots \alpha}{ }_{i}^{j}=\delta_{i}{ }^{j} K_{0}^{\mu \nu \ldots \alpha}
$$

In this case

$$
\begin{aligned}
& L=2 l, \quad\left(K_{0} n\right)=1, \\
& \left(K_{0} n\right)^{-1}=1, \quad\left(K_{0} n\right)^{\mu}=n^{\mu}, \\
& \left(K_{0} n\right)^{\mu \nu}=\frac{1}{2 l-1}\left(g^{\mu \nu}+(2 l-2) n^{\mu} n^{\nu}\right) .
\end{aligned}
$$

Using the rules formulated in the appendix B. it is easy to see that the result for the

nonminimal operator (65) gives the answer (41) for the minimal operator (15) in the flat space.

\section{E. The nonminimal vector field operator}

In a lot of papers one can encounter the following nonminimal second order operator

$$
D_{\alpha}^{\beta}=\delta_{\alpha}{ }^{\beta} \partial^{\mu} \partial_{\mu}-\lambda \partial_{\alpha} \partial^{\beta}+W_{\alpha}^{\beta}
$$

where 


$$
W_{\alpha \beta}=W_{\beta \alpha} .
$$

It can be written in the form (10) if

$$
K_{\alpha}^{\mu \nu}{ }^{\beta}=g^{\mu \nu} \delta_{\alpha}^{\beta}-\frac{\lambda}{2}\left(g^{\mu \beta} \delta_{\alpha}^{\nu}+g^{\nu \beta} \delta_{\alpha}^{\mu}\right)
$$

and hence

$$
\begin{aligned}
& (K n)_{\alpha}{ }^{\beta}=\delta_{\alpha}{ }^{\beta}-\lambda n_{\alpha} n^{\beta}, \\
& (K n)^{-1}{ }_{\alpha}{ }^{\beta}=\delta_{\alpha}{ }^{\beta}+\gamma n_{\alpha} n^{\beta}, \quad \text { where } \quad \gamma \equiv \frac{\lambda}{1-\lambda} .
\end{aligned}
$$

Substituting it to the equation (65) and using (68) we found that

$$
\begin{aligned}
& \Gamma_{\infty}^{(1)}=\frac{1}{16 \pi^{2}(d-4)} \operatorname{tr} \int d^{4} x \sqrt{-g}\left(\frac{1}{48} \gamma^{2} W^{2}\right. \\
& \left.+\left(\frac{1}{24} \gamma^{2}+\frac{1}{4} \gamma+\frac{1}{2}\right) W_{\mu \nu} W^{\mu \nu}\right)
\end{aligned}
$$

where $W \equiv W_{\alpha}{ }^{\alpha}$.

This expression is also in agreement with [7], [14] and [19].

\section{CONCLUSION.}

In this paper we presented a new method for making one-loop calculations in the nonminimal gauges [20], theories regularized by higher derivatives [21] and field theory models with higher spins.

¿From the mathematical point of view we found $a_{2}$ coefficient of the heat kernel expansion of an arbitrary differential operator without any restriction to its form and order. As a tool we choosed t'Hooft-Veltman diagramic approach with some modifications, in particular in the technick of calculating Feynman integrals.

Unfortunately, in the general case we can not use the classical gauge invariance that reliefs

calculations considerably for the minimal second order operator [22]. However we manage to obtain master formulas for the one-loop renormalization counterterms in theories with an arbitrary minimal operator in the curved space and an arbitrary nonminimal operator 
in the flat space. The consideration of some particular cases showed the agreement of our results with algorithms obtained earlier.

The result for a nonminimal operator can be generalized to the curved space. This will be reported in a separate paper. Of course, it is also possible to extend the method to multi-loop orders.

On the other hand, our approach can be considered as a step towards the total automatization of the one-loop divergences calculation. We also tries to create the appropriate software. For example, making calculation we often used our tensor package for the REDUCE system [23]. So, the most cumbersome operations in calculating one-loop Feynman diagrams now can be made by computers.

\section{APPENDIX A: CALCULATION OF THE DIVERGENT PART OF FEYNMAN INTEGRALS.}

Let us consider an integral

$$
\int d^{d} k f\left(k, p_{1}, p_{2} \ldots p_{n}\right)
$$

where $d$ is the space-time dimension.

Expanding $f$ in powers of $1 / k$ we have

$$
\begin{array}{r}
\int d^{d} k f\left(k, p_{1} \ldots p_{n}\right)=\int d^{d} k\left(\frac{1}{k^{(S)}} f_{S}\left(p_{1} \ldots p_{n}\right)\right. \\
\left.+\frac{1}{k^{(S+1)}} f_{S+1}\left(p_{1} \ldots p_{n}\right)+\ldots\right)
\end{array}
$$

Here $1 / k^{(S)}$ is an arbitrary expression built by $k_{\mu}$ of order $-S$, For example, by $1 / k^{(2)}$ we denote

$$
\frac{1}{k^{2}}, \frac{k_{\mu}}{k^{3}}, \frac{k_{\mu} k_{\nu}}{k^{4}} \text {, and so on }
$$

where $k=\sqrt{k^{2}}$.

The integrals are divergent if $s \leq 4$. In this case we first perform the angle integration. 
It is easy to see that

$$
\int d^{d} k \frac{1}{k^{L+S}} k_{\mu_{1}} k_{\mu_{2}} \ldots k_{\mu_{L}}
$$

is equal to 0 , if $L=2 l+1$ and

$$
\begin{aligned}
C \int d^{d} k \frac{1}{k^{S}} & \left(g_{\mu_{1} \mu_{2}} g_{\mu_{3} \mu_{4}} \ldots g_{\mu_{L-1} \mu_{L}}\right. \\
& \left.+g_{\mu_{1} \mu_{3}} g_{\mu_{2} \mu_{4}} \ldots g_{\mu_{L-1} \mu_{L}}+\ldots\right)
\end{aligned}
$$

if $L=2 l$

Here the sum is over all possible index replacements. It contains $(2 l-1) ! !=(2 l-1)(2 l-$ 3) $\ldots 1$ terms. $((-1) ! !=1)$ Contracting (A3) with $g_{\mu_{1} \mu_{2}} g_{\mu_{3} \mu_{4}} \ldots g_{\mu_{L-1} \mu_{L}}$ we found a constant C

$$
C=\frac{1}{2^{l}(l+1) !}
$$

Therefore, introducing a notation

$$
\begin{aligned}
& <n_{\mu_{1}} n_{\mu_{2}} \ldots n_{\mu_{2 l}}>\equiv \frac{1}{2^{l}(l+1) !} \\
& \times\left(g_{\mu_{1} \mu_{2}} g_{\mu_{3} \mu_{4}} \ldots g_{\mu_{2 l-1} \mu_{2 l}}\right. \\
& \left.\quad+g_{\mu_{1} \mu_{3}} g_{\mu_{2} \mu_{4}} \ldots g_{\mu_{2 l-1} \mu_{2 l}}+\ldots\right),
\end{aligned}
$$

we obtain

$$
\begin{aligned}
\int d^{d} k \frac{1}{k^{2 l+S}} & k_{\mu_{1}} k_{\mu_{2}} \ldots k_{\mu_{2 l}} \\
& =<n_{\mu_{1}} n_{\mu_{2}} \ldots n_{\mu_{2 l}}>\int d^{d} k \frac{1}{k^{S}}
\end{aligned}
$$

Using

$$
\int d^{d} k \frac{1}{\left(k^{2}+L\right)^{\alpha}}=\pi^{d / 2} e^{i \pi / 2-i \pi d / 2} \frac{\Gamma(\alpha-d / 2)}{\Gamma(\alpha)} L^{d / 2-\alpha}
$$

we conclude that nonzero result can be obtained if only $d / 2-\alpha=0$ (or $s=2 \alpha=4$ ). Then the divergent part will be the following: 


$$
\left(\int d^{d} k \frac{1}{k^{4}}\right)_{\infty}=\left(\lim _{L \rightarrow 0} \int d^{d} k \frac{1}{\left(k^{2}+L\right)^{2}}\right)_{\infty}=-\frac{2 i \pi^{2}}{d-4}
$$

and

$$
\begin{aligned}
&\left(\int d^{d} k\right.\left.\frac{1}{k^{2 l+S}} k_{\mu_{1}} k_{\mu_{2}} \ldots k_{\mu_{2 l}}\right)_{\infty} \\
&=-\frac{2 i \pi^{2}}{d-4}<n_{\mu_{1}} n_{\mu_{2}} \ldots n_{\mu_{2 l}}>
\end{aligned}
$$

Therefore, in order to calculate the divergent part of the integral (A1) we should expand $f$ in powers of $1 / k$, retain logarithmically divergent terms and perform the remaining integration by (A9).

\section{APPENDIX B: THE INTEGRATION OVER ANGLES FOR A MINIMAL OPERATOR}

Let us start with (A5):

$$
\begin{aligned}
& <n_{\mu_{1}} n_{\mu_{2}} \ldots n_{\mu_{2 m}}>\equiv \frac{1}{2^{m}(m+1) !} \\
& \times\left(g_{\mu_{1} \mu_{2}} g_{\mu_{3} \mu_{4}} \ldots g_{\mu_{2 m-1} \mu_{2 m}}\right. \\
& \left.\quad+g_{\mu_{1} \mu_{3}} g_{\mu_{2} \mu_{4}} \ldots g_{\mu_{2 m-1} \mu_{2 m}}+\ldots\right) .
\end{aligned}
$$

It can be interpreted in the following way: In order to obtain the result of the angle integration we should make pairs of $n_{\alpha}$ by all possible ways and add a numerical constant. Each pair of $n_{\alpha}$ and $n_{\beta}$ should be substituted by $g_{\alpha \beta}$.

The sum contains $(2 m-1)$ !! terms. Hence, if we contract (B1]) with a totally symmetric tensor $A^{\mu_{1} \mu_{2} \ldots \mu_{2 m}} \equiv A_{(2 m)}$ (here the bottom index points the tensor rank) the result will be

$$
<\left(A_{(2 m)} n\right)>=\frac{(2 m-1) ! !}{2^{m}(m+1) !} A,
$$

where $A \equiv A^{\mu_{1} \ldots \mu_{m}}{ }_{\mu_{1} \ldots \mu_{m}}$.

Similarly one can find that for a symmetric tensor $A_{(2 m-1)}$ with $2 m-1$ indexes the following equation takes place: 


$$
\begin{array}{r}
<n_{\alpha}\left(A_{(2 m-1)} n\right)>=\frac{(2 m-1) ! !}{2^{m}(m+1) !} A_{\alpha} \\
=\frac{2 m-1}{2(m+1)}<\left(A_{(2 m-1)} n\right)_{\alpha}>
\end{array}
$$

where $A_{\alpha} \equiv A^{\mu_{1} \ldots \mu_{m-1}}{ }_{\mu_{1} \ldots \mu_{m-1} \alpha}$.

In the more general case we will use the following consequence of (28):

$$
\begin{aligned}
& <n_{\mu_{1}} n_{\mu_{2}} \ldots n_{\mu_{2 m}}>=\frac{1}{2(m+1)}\left(g_{\mu_{1} \mu_{2}}<n_{\mu_{3}} n_{\mu_{4}} \ldots n_{\mu_{2 m}}>\right. \\
& \left.+g_{\mu_{1} \mu_{3}}<n_{\mu_{2}} n_{\mu_{4}} \ldots n_{\mu_{2 m}}>+\ldots+g_{\mu_{1} \mu_{2 m}}<n_{\mu_{2}} n_{\mu_{3}} \ldots n_{\mu_{2 m-1}}>\right) .
\end{aligned}
$$

Making contraction with 2 symmetric tensors we find that

$$
\begin{aligned}
<n_{\alpha}\left(A_{(2 m)} n\right) & \left(B_{(2 p-1)} n\right)>=\frac{1}{2(m+p+1)} \\
& \times\left(2 m<\left(A_{(2 m)} n\right)_{\alpha}\left(B_{(2 p-1)} n\right)>+(2 p-1)<\left(A_{(2 m)} n\right)\left(B_{(2 p-1)} n\right)_{\alpha}>\right) .
\end{aligned}
$$

This equation can be easily generalized to a greater number of symmetric tensors.

For a minimal operator we can explicitly make angle integration, although a result will be very cumbersome. Nevertheless, it is very convenient for computer calculations, because a number of operations to do becomes very small.

First we consider

$$
<\left(A_{(2 m)} n\right)\left(B_{(2 p)} n\right)>=\sum_{k=0}^{m} A^{\mu_{1} \ldots \mu_{2 k}} B_{\mu_{1} \ldots \mu_{2 k}} T_{m p}^{k}
$$

and assume that $m \leq p . T_{m p}^{k}$ is a numeric constant that should be calculated. It is easy to see, that

$$
T_{m p}^{k}=\frac{1}{2^{m+p}(m+p+1) !} C_{2 m}^{2 k}(2 m-2 k-1) ! !(2 p-2 k-1) ! ! \frac{(2 p) !}{(2 p-2 k) !} .
$$

Really, $1 / 2^{m+p}(m+p+1)$ ! is a normalization constant, $C_{2 m}^{2 k}$ gives a number of ways to choose $2 k n_{\alpha}$ from $\left(A_{(2 m)} n\right),(2 p) ! /(2 p-2 k)$ ! is a number of their possible combinations with $n_{\beta}$ from $\left(B_{(2 p)} n\right)$ and $(2 m-2 k-1) ! !(2 p-2 k-1)$ !! is a number of $n_{\mu}$ pairs inside $\left(A_{(2 m)} n\right)$ and $\left(B_{(2 p)} n\right)$.

By similar arguments 


$$
<\left(A_{(2 m+1)} n\right)\left(B_{(2 p+1)} n\right)>=\sum_{k=0}^{m} A^{\mu_{1} \ldots \mu_{2 k+1}} B_{\mu_{1} \ldots \mu_{2 k+1}} T_{m p}^{k}
$$

where

$$
T_{m p}^{k}=\frac{1}{2^{m+p+1}(m+p+2) !} C_{2 m+1}^{2 k+1}(2 m-2 k-1) ! !(2 p-2 k-1) ! ! \frac{(2 p+1) !}{(2 p-2 k) !}
$$

and $m \leq p$.

(B5) and (B8) can be written as

$$
<\left(A_{(m)} n\right)\left(B_{(p)} n\right)>=\sum_{k=0}^{m} A^{\mu_{1} \ldots \mu_{k}} B_{\mu_{1} \ldots \mu_{k}} T_{m p}^{k}
$$

where $m \leq p$ and

$$
T_{m p}^{k}=\frac{1}{2^{(m+p) / 2}((m+p) / 2+1) !} C_{m}^{k}(m-k-1) ! !(p-k-1) ! ! \frac{p !}{(p-k) !},
$$

if $m-k$ and $p-k$ are both even and 0 , otherwise.

This equation can be generalized to an arbitrary number of tensors. Here we consider cases that encounter in (52) and (54). Some terms there have a form

$$
\begin{aligned}
& <\left(A_{(2 l-2)} n\right)\left(B_{(2 l-1)} n\right)\left(C_{(2 l-1)} n\right)> \\
& =\sum_{m_{1}=0}^{2 l-2} \sum_{m_{2}=0}^{2 l-2} \sum_{m_{3}=0}^{2 l-1} A^{\mu_{1} \ldots \mu_{m_{1}} \nu_{1} \ldots \nu_{m_{2}}} B^{\mu_{1} \ldots \mu_{m_{1}} \alpha_{1} \ldots \alpha_{m_{3}}} C^{\nu_{1} \ldots \nu_{m_{2}} \alpha_{1} \ldots \alpha_{m_{3}}} T_{l}^{m_{1} m_{2} m_{3}} .
\end{aligned}
$$

By the help of the same method we can find that

$$
\begin{aligned}
& T_{l}^{m_{1} m_{2} m_{3}}=\frac{1}{2^{3 l-2}(3 l-1) !} C_{2 l-2}^{m_{1}} C_{2 l-2-m_{1}}^{m_{2}} C_{2 l-1-M}^{m_{3}}\left(2 l-2-m_{1}-m_{3}\right) ! ! \\
& \times\left(2 l-2-m_{2}-m_{3}\right) ! !\left(2 l-3-m_{1}-m_{2}\right) ! ! \frac{(2 l-1) !}{\left(2 l-1-m_{1}\right) !} \frac{(2 l-1) !}{\left(2 l-1-m_{2}\right) !} \frac{(2 l-1-m) !}{\left(2 l-1-m-m_{3}\right) !}
\end{aligned}
$$

if $m_{1}+m_{2}$ is even and $m_{1}+m_{3}, m_{2}+m_{3}$ is odd, otherwise $T_{l}^{m_{1} m_{2} m_{3}}=0\left(M \equiv \max \left(m_{1}, m_{2}\right)\right.$ $\left.m \equiv \min \left(m_{1}, m_{2}\right)\right)$.

A term $<\left(S_{(2 l-1)} n\right)^{4}>$ can be written as

$$
\begin{aligned}
& <\left(S_{(2 l-1)} n\right)^{4}>=\sum_{k_{1}=0}^{2 l-1} \sum_{k_{2}=0}^{2 l-1-k_{1}} \sum_{k_{3}=0}^{2 l-1-k_{1}-k_{2}} \sum_{k_{3}=0}^{2 l-1-M_{1}} \sum_{k_{3}=0}^{2 l-1-M_{2}} \sum_{k_{3}=0}^{2 l-1-M_{3}} \\
& S^{\mu_{1} \ldots \mu_{k_{1}} \nu_{1} \ldots \nu_{k_{2}} \sigma_{1} \ldots \sigma_{k_{3}}} S^{\mu_{1} \ldots \mu_{k_{1}} \alpha_{1} \ldots \alpha_{k_{4}} \beta_{1} \ldots \beta_{k_{5}}} S^{\nu_{1} \ldots \nu_{k_{2}} \alpha_{1} \ldots \alpha_{k_{4}} \gamma_{1} \ldots \gamma_{k_{6}}} \\
& \times S^{\sigma_{1} \ldots \sigma_{k_{3}} \beta_{1} \ldots \beta_{k_{5}} \gamma_{1} \ldots \gamma_{k_{6}}} T_{l}^{k_{1} k_{2} k_{3} k_{4} k_{5} k_{6}},
\end{aligned}
$$


where

$$
\begin{aligned}
& T_{l}^{k_{1} k_{2} k_{3} k_{4} k_{5} k_{6}}=\frac{1}{4^{2 l-1}(4 l-1) !}\left(2 l-2-k_{1}-k_{2}-k_{3}\right) ! ! \\
& \times\left(2 l-2-k_{1}-k_{4}-k_{5}\right) ! !\left(2 l-2-k_{2}-k_{4}-k_{6}\right) ! !\left(2 l-2-k_{3}-k_{5}-k_{6}\right) ! ! \\
& \times C_{2 l-1}^{k_{1}} \frac{(2 l-1) !}{\left(2 l-1-k_{1}\right) !} C_{2 l-1-k_{1}}^{k_{2}} \frac{(2 l-1) !}{\left(2 l-1-k_{2}\right) !} C_{2 l-1-k_{1}-k_{2}}^{k_{3}} \frac{(2 l-1) !}{\left(2 l-1-k_{3}\right) !} \\
& \times C_{2 l-1-M_{1}}^{k_{4}} \frac{\left(2 l-1-m_{1}\right) !}{\left(2 l-1-m_{1}-k_{4}\right) !} C_{2 l-1-M_{2}}^{k_{5}} \frac{\left(2 l-1-m_{2}\right) !}{\left(2 l-1-m_{2}-k_{5}\right) !} C_{2 l-1-M_{3}}^{k_{6}} \frac{\left(2 l-1-m_{3}\right) !}{\left(2 l-1-m_{3}-k_{5}\right) !},
\end{aligned}
$$

if $k_{1}+k_{2}+k_{3}, k_{1}+k_{4}+k_{5}, k_{2}+k_{4}+k_{6}, k_{3}+k_{5}+k_{6}$ are odd. Here

$$
\begin{array}{ll}
M_{1} \equiv \max \left(k_{1}, k_{2}\right), & m_{1} \equiv \min \left(k_{1}, k_{2}\right), \\
M_{2} \equiv \max \left(k_{1}+k_{4}, k_{3}\right), & m_{2} \equiv \min \left(k_{1}+k_{4}, k_{3}\right), \\
M_{3} \equiv \max \left(k_{2}+k_{4}, k_{3}+k_{5}\right), & m_{3} \equiv \min \left(k_{2}+k_{4}, k_{3}+k_{5}\right) .
\end{array}
$$

Otherwise

$$
T_{l}^{k_{1} k_{2} k_{3} k_{4} k_{5} k_{6}}=0
$$

We should note that rules formulated here are applicable only for a minimal operator. The matter is that for a nonminimal operator one can not present in general $(K n)^{-1}$ as a contraction of the form $(T n)$, where $T$ is a totally symmetric tensor. 


\section{REFERENCES}

[1] R.Jackiw, Phys.Rev. D 9, 1686, (1974).

[2] S.Minakshisundaram, J.Ind.Math.Soc 13, 41, (1949).

[3] R.T.Seeley, Amer.Math.Soc. 10, 288, (1967).

[4] P. B. Gilkey, J. Differ. Geomet. 10, 601, (1975); Duke Mathematical Journal 47, 511, (1980); Duke Mathematical Journal 48, 887, (1981).

[5] J.S.Schwinger, Phys. Rev. 82, 664, (1951).

[6] B.S.DeWitt, Dynamical Theory of Groups and Fields, (Gordon and Breach, Inc., New York, 1965).

[7] A. O. Barvinsky and G. A. Vilkovisky, Phys.Rep. 119, 1, (1985).

[8] L. F. Abbott, Nucl.Phys. B 185, 189, (1981).

[9] I. Jack and H. Osborn, Nucl.Phys. B 207, 474, (1982).

[10] Yu.M.Obukhov, Nucl.Phys. B 212, 237, (1983).

[11] N.H.Barth, J.Phys.A: Math.Gen., 20, 857, (1987); J.Phys.A: Math.Gen., 20, 875, (1987).

[12] G.Cognola and S.Zerbini, Phys.Lett. B 195, 435, (1987); Phys.Lett. B 214, 70, (1988).

[13] V. P. Gusynin, Nucl.Phys. B 333, 296, (1990).

[14] V.P.Gusynin, E.V.Gorbar and V.V.Romankov, Nucl.Phys. B 362, 449, (1991).

[15] C.Lee and C.Rim, Nucl.Phys. B 255, 439, (1985).

[16] I.Jack and H.Osborn, Nucl.Phys. B249, 472, (1985).

[17] A.O.Barvinsky, Yu.V.Gusev, G.A.Vilkovisky and V.V.Zhytnikov, Covariant Perturbation Theory (IV), Third Order in Curvature, Report of the University of Manitoba, Uni- 
versity of Manitoba, Winnipeg, (1993); J.Math.Phys., 35, 3525, (1994); J.Math.Phys., 35, 3543, (1994).

[18] G. t'Hooft and M. Veltman, Ann. Inst. Henri Poincaré 20, 69, (1974).

[19] E.S.Fradkin and A.A.Tseytlin, Higher Derivative Quantum Gravity: One-loop Counterterms and Asymptotic Freedom, preprint of P.Lebedev Physical Institute, N170, Moscow, (1981).

[20] R.E.Kallosh, O.V.Tarasov and I.V.Tyutin, Nucl.Phys. B 137, 145, (1978).

[21] A.A.Slavnov, Nucl.Phys. B31, 301, (1971).

[22] G. 't Hooft, Nucl.Phys. B 62, 444, (1973).

[23] P.I.Pronin and K.V.Stepanyantz, "New Tensor Package for REDUCE System", in New Computing Technick in Physics Research. IV., edited by B.Denby and D.Perred-Gallix, (World Scientific, Singapure, 263, 1995). 


\section{FIGURES}
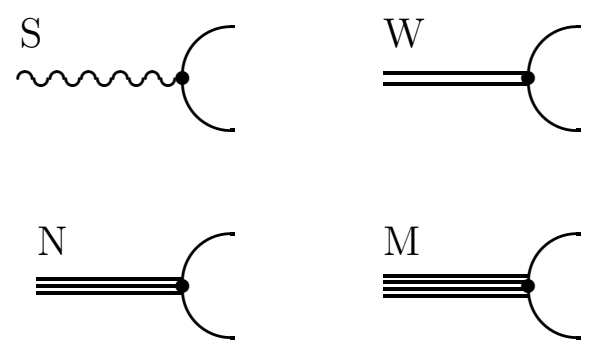

FIG. 1. Vertexes, that are encountered in the flat space divergent graphs.
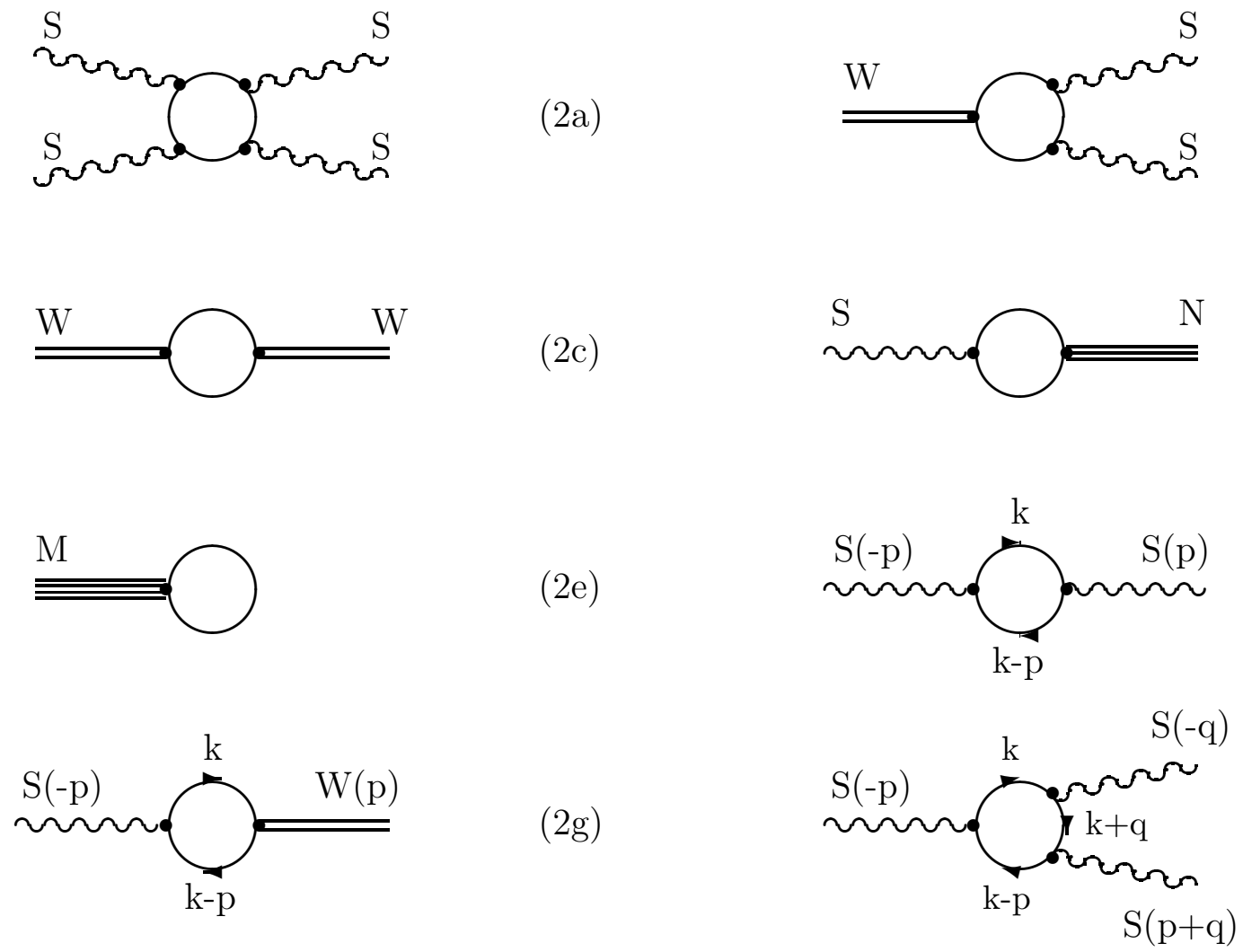

FIG. 2. Divergent graphs in the flat space. Diagrams (2a) - (2e) are logarithmically divergent, $(2 \mathrm{~g})$ and $(2 \mathrm{~h})$ are linearly divergent and $(2 \mathrm{f})$ is a quadratically divergent graph. 


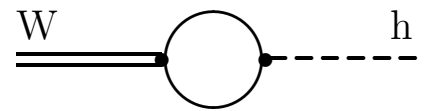

(3a)
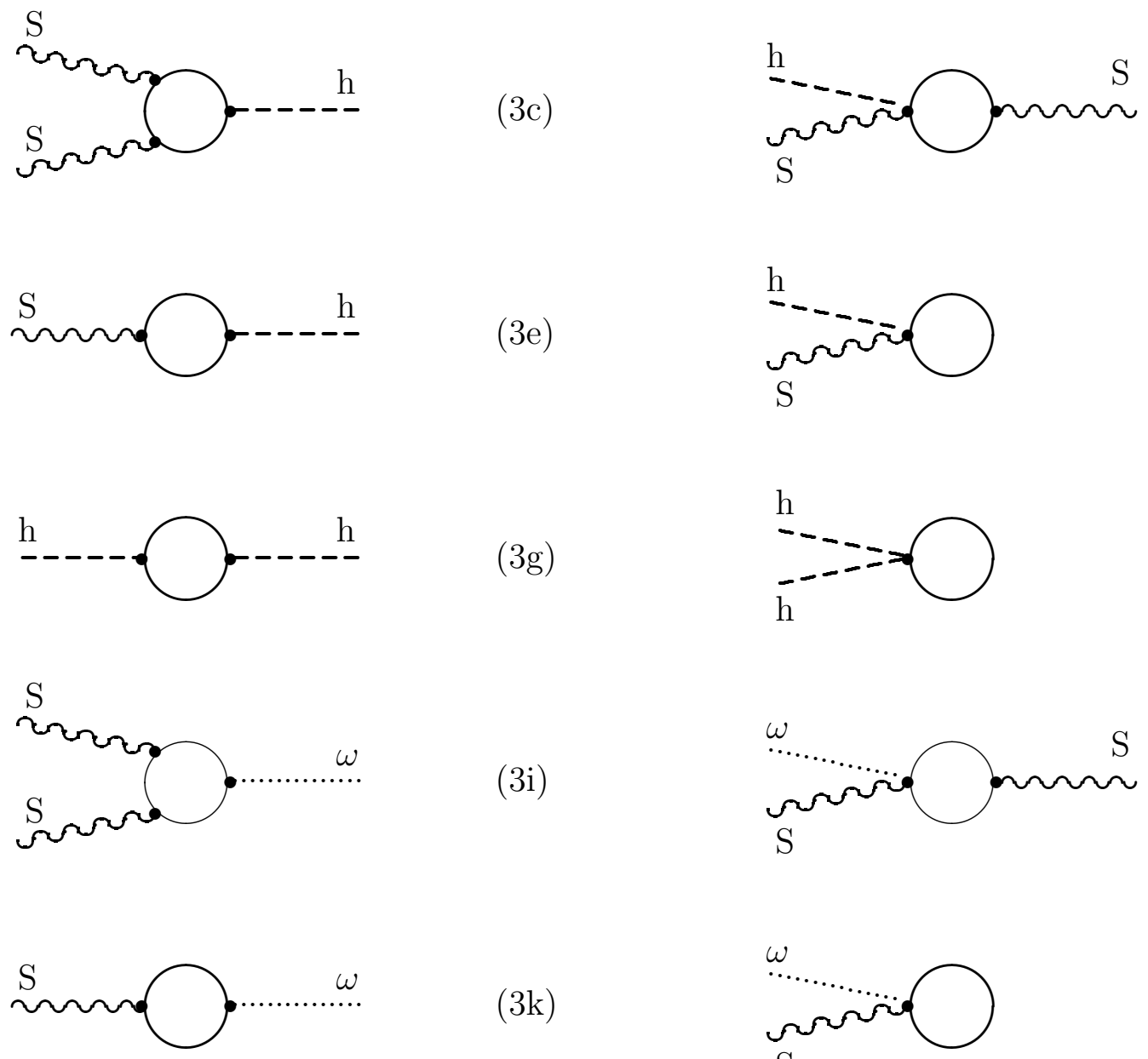

$\mathrm{S}$
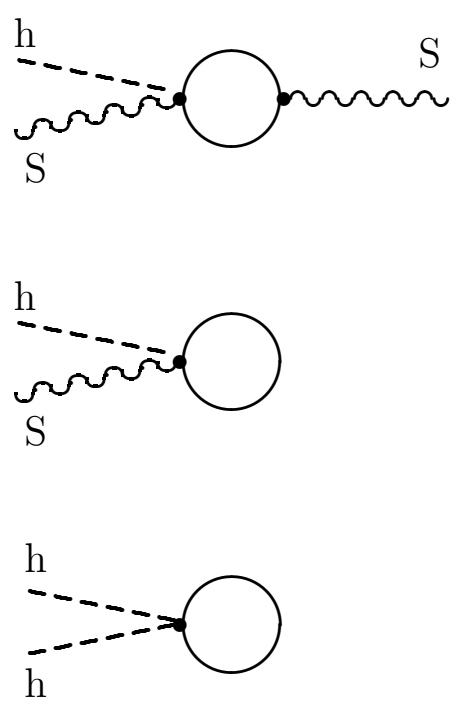
$\mathrm{S}$

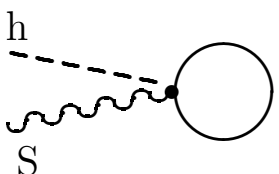

$\mathrm{W}$

$(3 \mathrm{~m})$

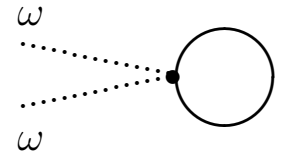

FIG. 3. Graphs for the effective action calculation in the curved space. 